\title{
Test unipodal para estabilidad corporal estática con ojos abiertos y cerrados en la valoración de riesgo de caída en adultos mayores Unipodal test for static body stability with open and closed eyes in the assessment of fall risk in older adults

\author{
*Cristian Díaz Escobar, **Macarena Catalán Neira, ***Matías Pozo Nuñez, ****Víctor Díaz Narváez \\ *Asociación Nacional de Fútbol Profesional (Chile), ${ }^{* *}$ Centro Médico Colina Salud (Chile), ${ }^{* * *}$ Universidad Bernardo \\ OHiggins (Chile), ${ }^{* * * *}$ Universidad Andres Bello (Chile)
}

\begin{abstract}
Resumen. Introducción: La estabilidad postural es un factor clave en la funcionalidad motora del ser humano, con el envejecimiento se produce alteración del balance postural y una alternativa de evaluación son las pruebas de estabilidad unipodal. Objetivo: Determinar el rendimiento de adultos mayores con el Test unipodal para estabilidad corporal estática (TUPECE) en modalidad ojos abiertos y ojos cerrados; observando estrategias de estabilización en pie de apoyo, movimiento de miembros superiores y pie elevado. Métodos: Estudio de concordancia diagnóstica, de corte transversal; realizado en 60 adultos mayores inscritos en un centro deportivo, comuna de San Miguel, Región Metropolitana, Chile. Quienes ejecutaron elTUPECE con ojos abiertos y cerrados, junto con una consulta referida al test. Resultados: La concordancia entre evaluadores en función al TUPECE no presentó diferencias significativas, demostrándose simetría en la evaluación observacional. Se determinó diferencia significativamente alta comparando la prueba con ojos abiertos y cerrados $(\mathrm{p}<0,0001)$, presentándose mayor estabilidad con ojos abiertos. A mayor edad la estabilidad es más deficiente $(\mathrm{p}<0.05)$. No hubo diferencia significativa entre géneros $(\mathrm{p}>0,05)$. Conclusión: El TUPECE presentó una alta confiabilidad interevaluador al valorar la estabilidad unipodal en adultos mayores. La prueba diferencia rendimiento con ojos abiertos y cerrados; demostró también diferencias por edad pero no por género. De acuerdo a los resultados y características de la prueba se expone como un procedimiento positivo para valorar la estabilidad unipodal estática con ojos abiertos y cerrados en adultos mayores.
\end{abstract}

Palabras claves: Estabilidad unipodal, adulto mayor, riesgo de caída.

\begin{abstract}
Introduction: Postural stability is a key factor in the motor functionality of the human being, with aging there is an alteration of the postural balance and an evaluation alternative is the unipodal stability tests. Objective: To determine the performance of older adults with the Unipodal Test for Static Body Stability (TUPECE) in open and closed eyes mode; observing stabilization strategies in standing support, movement of upper limbs and raised foot. Methods: Diagnostic concordance study, cross-sectional; conducted on 60 older adults enrolled in a sports center, San Miguel commune, Metropolitan Region, Chile. Those who executed the TUPECE with eyes open and closed, along with a consultation about the test. Results: The concordance between evaluators in theTUPECE did not present significant differences, demonstrating symmetry in the observational evaluation. High significant difference was determined comparing the test with open and closed eyes $(\mathrm{p}<0.0001)$, presenting greater stability with open eyes. The older the age, the poorer the stability $(\mathrm{p}<0.05)$. There was no significant difference between genders ( $\mathrm{p}>0.05)$. Conclusion:TUPECE presented a high interevaluator reliability when assessing unipodal stability in older adults. The test differentiates performance with open and closed eyes; it also showed differences by age but not by gender. According to the results and characteristics of the test, it is exposed as a positive procedure to evaluate the static unipodal stability with open and closed eyes in older adults.
\end{abstract}

Keywords: Unipodal stability, elderly, risk of falling.

\section{Introducción}

La postura, según Duclos et al. (2017) comprende uno de los soportes del movimiento humano, ajustando los segmentos corporales durante las transiciones desde una posición a otra de manera estable; donde el equilibrio es un estado y la estabilidad postural lo mantiene o

Fecha recepción: 25-12-20. Fecha de aceptación: 03-05-21

Víctor Patricio Díaz Narváez

vicpadina@gmail.com recupera sosteniendo la proyección vertical del centro de masa dentro de la base de sustentación del cuerpo. Para la mantención de la postura se requiere de un balance corporal mientras se realizan movimientos voluntarios o acciones reactivas en respuesta a perturbaciones externas (Osoba et al., 2019).

El control postural, se definiría como el acto de mantener, alcanzar o reestablecer un estado de equilibrio durante las posturas o acciones motrices de una persona (Cruz-Montecinos et al., 2020). Se le considera una habilidad motora compleja por la interacción que requie- 
re de múltiples procesos sensoriomotores, de los cuales se destacan el visual, vestibular y somatosensorial (Hong et al., 2016; Dunsky et al., 2017; Goel et al., 2017; Pelosin et al., 2018). Este conjunto de modalidades sensoriales permitiría identificar el espacio personal en relación al entorno, mediante la ubicación de los objetos, posiciones y desplazamientos del cuerpo, dando la configuración espacial de los segmentos corporales durante los movimientos (Foisy \& Kapoula, 2018).

En esta interacción de los sistemas para el control postural, el somatosensorial integraría la información propioceptiva de músculos y articulaciones, además de la información cutánea que identifica características de las superficies (Osoba et al., 2019); por ejemplo, la planta del pie informaría sobre la distribución de la presión plantar respecto a la referencia vertical del cuerpo durante desviaciones de la postura corporal (Foisy \& Kapoula, 2018). Mientras que el tríceps sural influiría en el control de las oscilaciones presentadas durante la mantención de la posición bípeda; en este trabajo integrado, la información plantar tendría mayor relevancia durante oscilaciones corporales leves y la propioceptiva de tobillo, donde se integra el tríceps sural, en las oscilaciones de mayor amplitud (Duclos et al., 2017). Por su parte, durante el control postural el sistema vestibular integraría la información relacionada con la orientación de la cabeza y tronco en el espacio, permitiendo la detección de la posición y dirección de los movimientos corporales (Osoba et al., 2019; Dunsky, 2019). A su vez, el sistema visual aportaría mediante la información proveniente de la sensibilidad al contraste, agudeza visual, percepción de profundidad y restricción del campo visual (Riva et al., 2019). Si bien, los tres sistemas tendrían relevancia en el control postural, para Donoso et al. (2019), cuando existe dependencia visual habría una mayor priorización de esta información por sobre los otros sistemas; lo que podría manifestarse durante la evaluación de la estabilidad postural si la persona presenta dificultad para sostener la posición erguida mientras se le solicita mantener los ojos cerrados.

El envejecimiento es un proceso multifactorial que involucra variables genéticas, biológicas y socio-ambientales; con el tiempo, la interacción de estas variables se afecta provocando deterioro en funciones corporales y motoras como la coordinación o el equilibrio, incrementando en riesgo de caída en los adultos mayores (AM) (Saüch etal., 2015; Puszczalowska et al., 2018, Navalón \& Martínez, 2020). Las caídas son la causa más común de pérdida de independencia, declive funcional e incremento de la mortalidad en los AM (Pua et al.,
2017; Marmamula et al., 2020). Exponiéndose como uno de los principales problemas en salud pública, por el costo que esto significa para los países y el deterioro que provoca en la calidad de vida de esta población (Sampedro et al., 2015; Olivera et al., 2018).

El riesgo de caída en los AM está asociado a variados factores de tipo individual como ambiental, en el caso de los factores intrínsecos con mayor asociación al presente estudio se destacan la edad, género, atrofia muscular, deterioro de la visión y también del balance corporal tanto estático como dinámico (Puszczalowska-Lizis et al., 2018; Marmamula et al., 2020; Cohen et al., 2020). La afectación del balance sería producto de la alteración en los tiempos de reacción que se requiere para mantener el equilibrio postural durante las acciones motoras (Mesquita et al., 2015). Esto último, considerado una variable clave en la valoración del control postural y riesgo de caída de los AM mediante pruebas de tipo estáticas y dinámicas (Dunsky et al., 2017).

En el ambiente clínico, la evaluación de la estabilidad dinámica se realiza principalmente con el Timed Up and Go Test y en la estabilidad estática a través de pruebas unipodales con ojos abiertos y cerrados. En laboratorio las intervenciones serían más específicas con la aplicación de plataformas de fuerza o acelerómetros tridimensionales (Lee \& Sun, 2018). En la evaluación de la función postural, Paillard \& Noé (2015), manifiestan la importancia de tener materiales tecnológicos pero también pruebas simples como indicadores generales de la eficiencia en el control postural.

Parte de la funcionalidad motora, relacionada con el control postural, esta asociada al balance unipodal presente en variadas actividades de la vida diaria como: la marcha, al sortear obstáculos o subir escaleras, entre otras (Bonora et al., 2017) y su valoración puede ser a través de la prueba de equilibrio unipodal estático, descrita como un método sencillo, de mínimos requerimientos en recursos, capacitación simple y aplicable en variados entornos para determinar el grado de fragilidad, autonomía y riesgo de caída de los AM (Springer et al., 2007). Sin embargo, en atención a las consideraciones expresadas por Paillard \& Noé (2015) respecto a que los métodos y técnicas de evaluación deben proporcionar variables cuantitativas y cualitativas confiables, especialmente en la valoración de los estados funcionales. Para la evaluación del balance corporal, debiese tenerse presente algunos aspectos esenciales, como los expuestos por Duclos et al. (2017), quienes explican que las sinergias musculares en la reorganización postural rápida, frente a la pérdida del balance tendría tres es- 
trategias principales: comenzando por tobillo, seguido de cadera y por último la iniciación del paso para lograr la estabilización. Este tipo de estrategia correctora frente a la desestabilización postural se iría modificando en función a la edad y el objetivo del movimiento; exponiendo como ejemplo que los AM tendrían una tendencia mayor a utilizar el desplazamiento de los miembros superiores o varios pasos cortos cuando pierden la estabilidad en comparación a personas más jóvenes (Duclos et al., 2017). Antecedentes que argumentarían la importancia de considerar el accionar de los miembros superiores en estrategias de estabilización, especialmente por parte de los AM, durante el control de la estabilidad corporal, en conjunto con los cambios que también van afectando a los miembros inferiores, donde según Kim \& Park (2016), con los años la interacción de la información proveniente de todo el segmento corporal, especialmente desde tobillo, rodilla y cadera, para lograr un eficiente control del balance se vuelve más complejo; generándose por ejemplo mayor oscilación corporal en los AM, incluso durante la mantención de posturas sencillas como la posición bípeda (Mesquita et al., 2015). Cambios que se van produciendo por la disminución de la conciencia respecto a la posición de los pies durante los movimientos producto de la pérdida de información propioceptiva desde la zona pie-tobillo y aumento de la coactivación de la musculatura de esta área, provocando estrategias posturales más rígidas para lograr mantener la estabilidad (Yamagat et al., 2017; Osaba et al., 2019). Sumado a las alteraciones que también comienzan a manifestarse en el sistema vestibular y visual; este último con una influencia crítica para la posición bípeda como unipodal por su influencia en el incremento del riesgo de caída en los AM (Saftari \& Kwon, 2018).

De acuerdo a lo expuesto, el presente estudio tuvo como objetivo principal comparar el comportamiento de un grupo de AM con el método denominado test unipodal para estabilidad corporal estática (TUPECE), observando estrategias de estabilización a nivel de miembros superiores e inferiores con ojos abiertos y cerrados durante la postura unipodal. Entendiéndose que las estrategias de estabilización serían diversas y pueden servir como diagnóstico diferencial para cada individuo, especialmente en AM frente al riesgo de caída.

\section{Material y método}

Estudio descriptivo, de corte transversal; consistente en determinar la concordancia diagnóstica entre dos evaluadores aplicando una evaluación de estabilidad corporal denominado Test unipodal para estabilidad corporal estática (TUPECE) con ojos abiertos y cerrados en un grupo de AM; y el comportamiento del grupo en la valoración del equilibrio unipodal estático con y sin el uso de la visión. El procedimiento se realizó el mes de Febrero año 2020 con una muestra de 60 AM autovalentes (43 mujeres, media en edad: 68,95 + 5,73 años -17 hombres, media en edad: 72,71 + 6,15 años) inscritos en un centro deportivo municipal de la comuna de San Miguel, Región Metropolitana, Chile.

Criterio de inclusión: sujetos de 60 o más años, con deambulación autónoma sin uso de aparatos de asistencia, con capacidad para comprender y seguir instrucciones sencillas, tener enfermedades crónicas clínicamente controladas y en la fecha de evaluación no cursar con algún tipo de condición aguda como: estado gripal, cefalea o dolencia física que comprometiera su desempeño durante la evaluación; firmar el consentimiento informado.

Criterio de exclusión: retiro voluntario por alguna razón de tipo física o psicológica que se produjera durante el proceso; sin ningún tipo de compromiso o explicación por parte de la persona, si así lo determinara, hacia los investigadores.

La confiabilidad del instrumento fue estimada mediante la prueba á de Cronbach. Además, el instrumento TUPECE fue sometido a un estudio de validación de constructo en estabilidad unipodal mediante Criterio de Jueces. Los jueces estuvieron constituidos por dos kinesiólogos especialistas en movimiento, uno deportivo y dos de rehabilitación kinésica.

En un espacio habilitado en el centro deportivo, los AM fueron citados en grupos de cinco o seis personas; se les solicitó permanecer sentados mientras se completaba la ficha personal. Posteriormente de manera individual pasaron a un área aislada para evaluación del TUPECE.

Como procedimiento del TUPECE se establece: sujeto ubicado en posición bípeda, pasando posteriormente a posición unipodal sobre una cruz de 20 centímetros marcada en el piso con cinta adhesiva de 2 centímetros de ancho, existiendo contraste entre el color de la cinta y el piso; manteniendo los brazos relajados al costado del tronco y la mirada hacia el frente (Figura 1). La prueba inicia con el pie derecho sobre la cruz, a la indicación del evaluador la persona eleva el pie contrario generando una flexión de cadera y rodilla (sin ser obligatorio lograr $90^{\circ}$ en ambas articulaciones), la posición se debe mantener un máximo de 10 segundos; si el 
tiempo se cumple se indica descanso volviendo a posición bípeda; para posteriormente repetir el procedimiento cambiando el pie de apoyo. En total se realizan 8 ejecuciones, alternando apoyo pie derecho-izquierdo primero con ojos abiertos y después con ojos cerrados. La evaluación consigna movimiento de manos o pie de apoyo por cada intento. En manos, si no se produce movimientos $=0$ punto, si una o ambas al mismo tiempo se mueven $1 \mathrm{vez}=1$ punto, 2 o más movimientos de una o ambas manos $=2$ puntos. En pie de apoyo: $\sin$ movimiento $=0$ punto, pie se mueve pero manteniendo contacto sobre la cruz $=1$ punto, si el pie se desplaza y deja de tocar la cruz $=2$ puntos. Si el pie elevado toca el piso se detiene el tiempo para ese intento consignándose 4 puntos (2 en manos -2 en pie), prosiguiéndose con la siguiente ejecución hasta completar los 8 intentos.

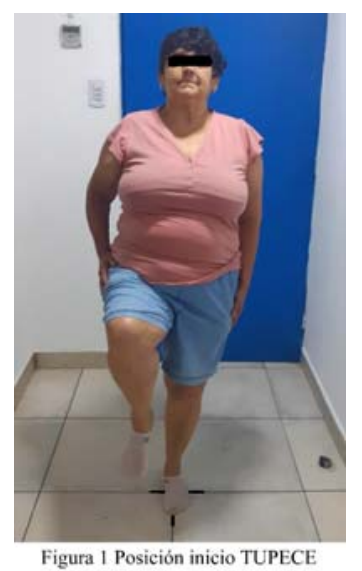

Se registra un puntaje mínimo de 0 punto y un máximo de 8 puntos, sumándose las dos ejecuciones correspondientes a pie derecho ojos abiertos y pie izquierdo ojos abiertos, lo mismo se realiza para los intentos con ojos cerrados. Estableciéndose que la persona presenta buen equilibrio con un puntaje igual o menor a 4 puntos y equilibrio deficiente con puntaje de 5 o superior, tanto para ojos abiertos como cerrados según lado derecho e izquierdo. Durante el proceso un kinesiólogo debidamente entrenado, anexo al grupo de investigación, se mantuvo próximo a los sujetos durante la evaluación en caso que requirieran algún tipo de asistencia. Al final de la evaluación el evaluado respondió el instrumento de consulta referido al TUPECE.

El proyecto respetó los principios bioéticos en investigación científica por lo que fue aprobado por el Comité de Ética de la Universidad Europea del Atlántico (España) con número de registro CLSNMGS2858691 (Enero 2020).

\section{Análisis estadístico}

Los datos observados por los dos operadores que realizaron el procedimiento fueron sometidos a estudios de concordancia mediante el estadístico Kappa de Cohen y fue calculado el error estándar asintótico. Adicionalmente, fue estimado el estadístico T con significación aproximada y exacta. Fue utilizada la prueba de proporciones para dos muestras independientes para comparar las proporciones de pacientes con resultados estables e inestables. La prueba t-student fue empleada para determinar diferencias entre las medias de los años en sujetos estables e inestables en diferentes condiciones de medición. Por último, la asociación entre el género y la condición de estabilidad o no estabilidad en diferentes condiciones de medición fue estimada mediante la prueba de chi-cuadrada. El nivel de significación empleado fue de ád»0,05.

\section{Resultados}

Los resultados de la estimación de la concordancia entre los evaluadores en relación con la estabilidad e inestabilidad de los sujetos evaluados en diferentes condiciones se presentan en la Tabla 1. Se observó que no existen diferencias significativas entre los observados y la prueba estadística demostró simetría en las observaciones realizadas por ambos evaluadores.

Los resultados de la comparación de la estabilidad entre los AM con ojos abiertos y cerrados con pie derecho y entre AM con los ojos abiertos y cerrados con pie izquierdo se presentan en la Tabla 2. Se observó que existen diferencias altamente significativas $(p<0,0001)$ entre los grupos comparados, favoreciendo a los sujetos estables que fueron medidos con los ojos abiertos, independientemente del pie usado en la medición.

En la Tabla 3 se presentan los resultados de la estimación de las medias de las edades entre sujetos estables e inestables en AM con ojos abiertos y cerrados con pie derecho y entre AM con los ojos abiertos y cerrados con pie izquierdo. En todos los casos se observaron diferencia significativas $(\mathrm{p}<0,05)$ y altamente significativas $(p<0,005)$ entre las medias de las edades de los sujetos con estabilidad en relación con aquellos que no la tienen. En todos los casos la media de los AM estables fue menor que la de los sujetos inestables.

En la Tabla 4 se presenta la asociación entre el género y las diferentes condiciones en que se midió la estabilidad de los AM. Se observó que la prueba de chicuadrado no fue significativa $(\mathrm{p}>0,05)$ en todos los casos. Como consecuencia, el género es independiente de 
la medición de estabilidad en las condiciones estudiadas.

En la consulta al grupo de estudio respecto a la aplicación del TUPECE: El 91,7\% de las personas percibió la prueba como no peligrosa para la integridad física, el $100 \%$ manifestó que la prueba no le generó cansancio, el 88,3\% indicó que las instrucciones fueron fáciles de entender, al 93,3\% le gustó la prueba y el 100\% refirió que repetiría la prueba si se evaluara nuevamente estabilidad unipodal estática.

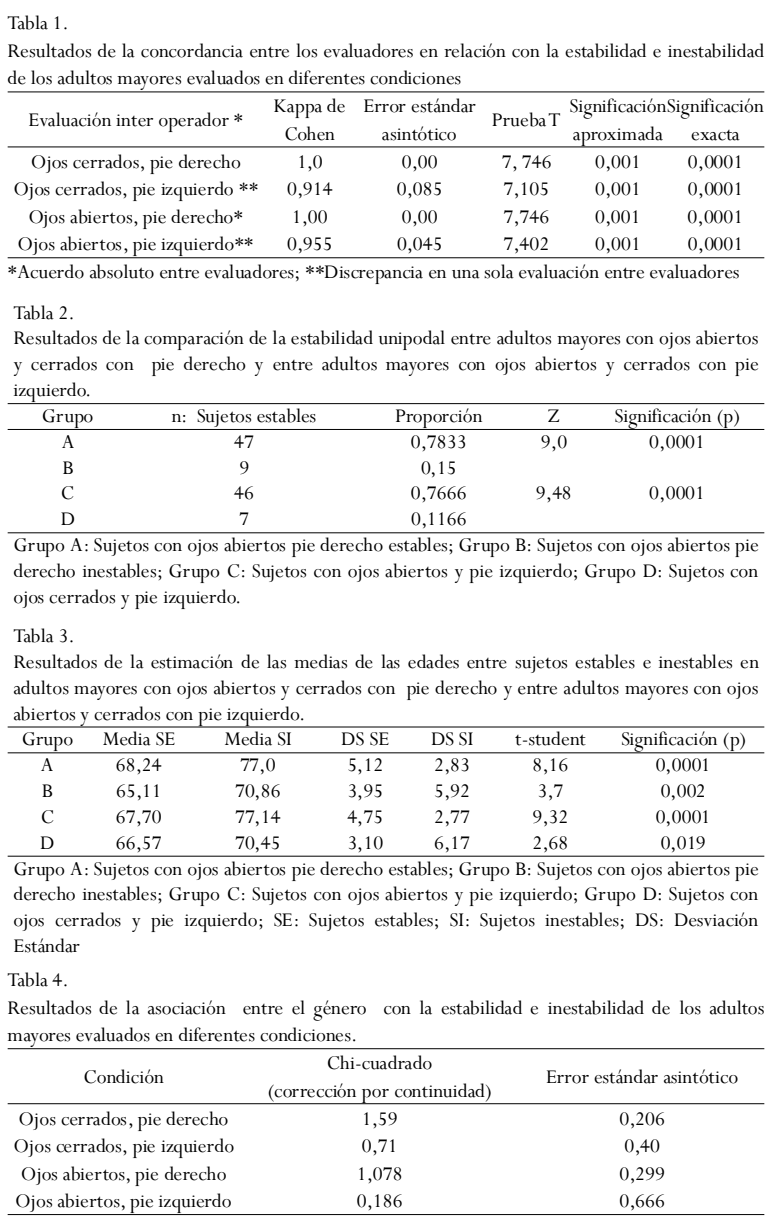

\section{Discusión}

Las sociedades actuales presentan una transición demográfica sin precedentes, con un incremento exponencial de la población AM, a quienes la calidad de vida se les va deteriorando, en parte, por la alteración de su funcionalidad. Lo que no significa que la persona simplemente se resigne y acepte esta pérdida o detrimento de su salud; por el contrario, debiera existir un aprendizaje de cómo conservar la calidad de vida mediante el autocuidado y adecuadas pautas de vida (Salazar et al., 2015). En los sistemas de salud, la calidad de vida de los AM es un tema prioritario, aplicándose estrategias preventivas a través de procesos multidisciplinarios y controles periódicos. Sin embargo, la posibilidad de utilizar pero también aportar nuevos conocimientos en esta materia, debiese ser una constante en los profesionales de la salud mejorando o proponiendo nuevas estrategias de intervención que optimicen los recursos o procedimientos mejorando la calidad de vida de esta población. Aspecto que fundamentaría parte del propósito del presente estudio con enfoque en la evaluación de la estabilidad postural estática con ojos abiertos y cerrados en un grupo de AM por su relación con un síndrome geriátrico de gran importancia como son las caídas y sus consecuencias.

La estabilidad postural tiene un papel relevante en la motricidad e independencia del ser humano. Durante las actividades de la vida diaria las posturas corporales van cambiando constantemente, requiriéndose alternancia entre el control postural estático, dinámico y de reacción (Springer et al., 2007). Con el envejecimiento como proceso natural e irreversible se provoca deterioro en las funciones corporales (Puszczalowska-Lizis et al., 2018) y una de ellas es el balance corporal que a su vez tiene una condición multifactorial. Por la relación existente entre balance postural y riesgo de caída en AM; Hong et al. (2016), expusieron la necesidad de contar con un método fácil para la valoración de la estabilidad postural. No obstante, Duclos et al. (2017) manifestaron que por la complejidad que tiene el control motor, en relación al balance corporal, se dificulta una prueba simple que sea lo suficientemente completa para lograr este objetivo. Recomendándose por parte de Toosizadeh et al. (2018) enfocar los esfuerzos primero en identificar factores de riesgo relacionados con el deterioro de un componente en específico o la interacción deficiente entre las variables, que en este caso, conforman el balance corporal. Aspecto que se considera en las evaluaciones preventivas anuales en AM por parte del Ministerio de Salud Chileno, consignando en el caso del balance corporal y riesgo de caída el Timed Up and Go Test para el balance dinámico y el test Estación Unipodal para el balance estático. Evaluaciones ajustadas a los objetivos requeridos en la prevención de caídas de los AM; sin embargo, Mancilla et al. (2015) expusieron la necesidad de continuar mejorando la valoración de la funcionalidad de los AM debido al acelerado envejecimiento poblacional que se está presentando en el país.

Frente a la problemática del envejecimiento y desbalance corporal, la investigación clínica enfocada en 
la postura unipodal se plantea como buena alternativa, por la complejidad que representa para el ser humano esta posición (Semak et al., 2020). En este requerimiento de optimizar intervenciones clínicas, surge como alternativa el TUPECE por la relación existente entre estabilidad postural y riesgo de caída del AM, exponiendo los siguientes fundamentos: a) riesgo de caída y tiempo: se ha indicado que los AM que presentan una estabilidad unipodal menor a 5 segundos tienen 2,5 veces más riesgo de sufrir una caída (Springer et al., 2007). Por su parte, Mancilla et al. (2015), indicaron que a mayor edad menor tiempo de permanencia en la posición unipodal y con tiempo menor o igual a 5 segundos el riesgo de caída se incrementa. Por tanto, para elTUPECE se determinó 10 segundos por intento considerando como media los 5 segundos referenciados por la literatura científica en cuanto a mayor o menor riesgo de caída. No obstante, la buena o deficiente estabilidad unipodal para la prueba se establece según puntaje, con una relación inversa respecto al tiempo en los otros métodos, determinándose 4 o menos puntos para buena estabilidad y sobre 4 puntos estabilidad deficiente en un máximo de 8 puntos para ojos abiertos y ojos cerrados, según pierna derecha o pierna izquierda; b) Tiempo de ejecución y fatiga muscular o cansancio: la prueba de Estación Unipodal, aplicada en Chile, solicita mantener la posición con un máximo de 30 segundos por intento, con tres ejecuciones por cada miembro inferior; además de la mantención de la extremidad libre con flexión de cadera y rodilla en 90 grados (Mancilla et al., 2015). En el caso del TUPECE, al consignar un tiempo máximo de 10 segundos en las 8 ejecuciones requeridas (4 por cada miembro inferior) y el no exigir que la extremidad superior mantenga los ángulos de flexión de la prueba; se intenta prevenir una mayor posibilidad de fatiga muscular en los miembros inferiores al establecer que con un máximo de 10 segundos en la posición unipodal se puede establecer buena o deficiente estabilidad postural por parte de la persona y que el tiempo total de la prueba, no sea un factor de cansancio, esperando que su duración total incluyendo explicaciones y cambios de posición no supere los 3 minutos por persona. Condición que se sustentó en la consulta sobre el instrumento a los AM, quienes en un 100\% manifestaron que la prueba no les generó cansancio.

El puntaje para TUPECE está basado en la observación de estrategias de estabilización postural, a nivel de pie-tobillo por ser un área con mayor pérdida propioceptiva por parte de los AM (Osaba et al., 2019) y movimiento de miembros superiores, por ser referi- da como estrategia más utilizada por esta población en comparación a sujetos más jóvenes (Duclos et al., 2017). Junto al apoyo del pie elevado, como acción en busca de ejecutar un paso cuando se produce una inestabilidad importante (Duclos et al., 2017). Elementos observacionales que a través de la concordancia entre evaluadores en la valoración de estabilidad o inestabilidad unipodal de los sujetos, demostró simetría en los resultados, lo que permite postular una buena confiabilidad del instrumento.

En cuanto a la evaluación con ojos abiertos y cerrados, tiene sustento por la importancia que se le atribuye a la visión en el control de la estabilidad corporal (Kim \& Park, 2016; Saftari \& Kwon, 2018). No obstante, la evaluación para estabilidad unipodal en AM aplicada en la salud pública chilena sólo consiga la evaluación con ojos abiertos (Mancilla et al., 2015). Condición que ya tendría una diferencia respecto al TUPECE, método con el cual se determinó diferencias significativas en un grupo de AM durante el rendimiento unipodal con ojos abiertos y cerrados. Resultados que podrían sumar nuevos aportes en la determinación de dependencia visual de esta población; especialmente por su implicancia en la capacidad para adaptar estrategias de control postural y riesgo de caída frente a nuevos entornos sensoriomotores a los cuales se enfrenta esta población (Springer et al., 2007; Goel et al., 2017), situación que actualmente no se consideraría en la evaluación preventiva del AM, propuesta por el Ministerio de Salud en Chile.

A su vez, los resultados delTUPECE, en lo general, permiten la valoración individual o tendencia grupal como rendimiento de la estabilidad unipodal con ojos abiertos y cerrados (buena estabilidad o estabilidad deficiente). Pero también en lo específico puede analizarse individualmente el tipo de estrategia utilizada en cuanto a uso del pie de base, miembros superiores o apoyo del pie elevado; y si esto tiene un comportamiento similar entre la primera y segunda parte de la prueba, lo que podría estar relacionado con un componente de aprendizaje o incremento de la seguridad personal ante el procedimiento.

Respecto a la edad se ratifica lo expuesto por Springer et al. (2007) presentándose diferencias significativas en el grupo, con incremento de la inestabilidad unipodal en edades más avanzadas. No así en el análisis de género, donde pudo influir el tamaño de la muestra, aunque un resultado similar fue presentado por Marmamula et al. (2020). Antecedente que también pueden resultar interesantes si con el tiempo se lograr determinar tipos 
de estrategias de estabilización de los segmentos corporales de acuerdo a la edad.

Además la prueba fue percibida por la mayoría del grupo como un procedimiento que no representaba riesgo para la integridad física, con instrucciones fáciles de comprender y la totalidad de los AM repetiría el TUPECE si se evaluara nuevamente la estabilidad unipodal estática; además, este procedimiento presentaría características similares a otros métodos en cuanto a mínimos recursos para su aplicación, fácil ejecución, capacitación simple, entre otras (Springer et al., 2007; Bonora et al., 2017). Características que en su conjunto se exponen como positivas en la valoración del riesgo de caída de los AM. Entendiéndose que un instrumento por sí sólo no puede contener todas las variables implícitas en la valoración funcional del AM. No obstante, mientras más información se proporcione en esta materia mayor posibilidad de desarrollar mejores estrategias de intervención en el área preventiva geriátrica, especialmente a nivel de sistema público.

Como limitaciones del estudio, referido a la utilización del TUPECE en un grupo de AM para evaluar la estabilidad unipodal con ojos abiertos y cerrados, en relación al riesgo de caída de esta población se plantea: a) gestionar la aplicación de la prueba a una muestra mayor o grupos con condiciones clínicas específicas, por ejemplo, adultos mayores con alteración vestibular o visual diagnosticada, institucionalizados, rehabilitación post artroplastia, entre otros; b) consignar además del puntaje por intento si la persona mantiene la posición unipodal por sobre o bajos los 5 segundos en relación a mayor o menor riesgo de caída; c) si se consignara la posibilidad de registrar en video la prueba, sumando este costo como recurso, se podrían analizar con mayor objetividad las estrategias de estabilización utilizadas por las personas analizando lo que ocurre con el pie de apoyo, el pie elevado y movimientos de miembros superiores, el uso de posibles movimientos a nivel de cadera-tronco; como también el comportamiento de la posición del miembro inferior elevado durante todo el procedimiento por su posible relación con la atrofia muscular asociada al envejecimiento; d) realizar intervenciones comparando elTUPECE con un gold estándar, por ejemplo posturografía en laboratorio oTest de Estación Unipodal como prueba de campo. Variables que a futuro pueden representar más elementos positivos para este procedimiento, sumándose a los expuestos en el presente estudio como aporte a la prevención en salud geriátrica especialmente en la valoración del riesgo de caída de esta población.

\section{Conclusión}

El Test unipodal para estabilidad corporal estática (TUPECE) con ojos abiertos y cerrados en un grupo de adultos mayores autovalentes presentó alta confiabilidad interevaluador, diferencias significativas al comparar rendimiento con ojos abiertos y cerrados; estabilidad deficiente a medida que aumenta la edad y sin diferencias al ser comparada entre géneros. Como primera intervención también se destaca, en cuanto a características de la prueba, tener un procedimiento y ejecución simple, ser de bajo costo y entrega información tanto grupal como individual aplicable a estrategias de control o prevención en el área clínica preventiva geriátrica.

\section{Referencias}

Bonora, G., Mancini, M., Carpinella, I., Chiari, L., Ferrarin, M., Nutt, J. G., \& Horak, F. B. (2017). Investigation of Anticipatory Postural Adjustments during One-Leg Stance Using Inertial Sensors: Evidence from Subjects with Parkinsonism. Frontiers in neurology, 8, 361. https://doi.org/10.3389/ fneur.2017.00361

Cohen, R. G., Baer, J.L., Ravichandra, R., Kral, D., McGowan, C., \& Cacciatore, T.W. (2020). Lighten Up! Postural Instructions Affect Static and Dynamic Balance in Healthy Older Adults. Innovation in aging, 4(2), igz056. https://doi.org/ 10.1093/geroni/igz056

Cruz-Montecinos, C., Cuesta-Vargas, A., Muñoz, C., Flores, D., Ellsworth, J., Fuente, C., Calatayud, J., Rivera-Lillo, G., SotoArellano, V., Tapia, C., \& García-Massó, X. (2020). Impact of Visual Biofeedback of Trunk Sway Smoothness on Motor Learning during Unipedal Stance. Sensors (Basel, Switzerland), 20(9), 2585. https://doi.org/10.3390/ s20092585

DonosoT, Silvia, Maulén C,Valentina, \& Novoa C, Ignacio. (2019). Dependencia visual en otoneurología: Consideraciones clínicas para la rehabilitación vestibular. Revista de otorrinolaringología y cirugía de cabeza y cuello, 79(3), 374-380. https: / / dx.doi.org/10.4067/S0718-48162019000300374

Duclos, N., Duclos, C., \& Mesure S. (2017). Control postural: fisiología, conceptos principales e implicaciones para la readaptación. EMC - Kinesiterapia - Medicina Física, 38(2), 1-9. http: / /dx.doi.org/10.1016/S1293-2965(17)83662-8

Dunsky A. (2019). The Effect of Balance and Coordination Exercises on Quality of Life in Older Adults: A MiniReview. Frontiers in aging neuroscience, 11, 318. https:// doi.org/10.3389/fnagi.2019.00318

Dunsky, A., Zeev, A., \& Netz,Y. (2017). Balance Performance Is Task Specific in Older Adults. BioMed research international, 2017, 6987017. https://doi.org/10.1155/ 2017/6987017 
Foisy,A., \& Kapoula,Z.(2018). Plantar cutaneous afferentsinfluence the perception of Subjective Visual Vertical in quiet stance. Scientific reports, 8(1), 14939. https://doi.org/ 10.1038/s41598-018-33268-3

Goel, R., DeDios,Y.E., Gadd, N.E., Caldwell,E.E., Peters, B.T., Reschke, M.F., Bloomberg, J. J., Oddsson, L., \& Mulavara,A. P. (2017). Assessing Somatosensory Utilization during Unipedal Postural Control. Frontiers in systems neuroscience, 11,21.https: / /doi.org/10.3389/fnsys.2017.00021

Hong, C.Y., Guo, L.Y., Song, R., Nagurka, M. L., Sung, J. L., \&Yen, C.W. (2016). Assessing postural stability via the correlation patterns of vertical ground reaction force components. Biomedical engineering online, 15(1), 90. https:/ /doi.org/10.1186/s12938-016-0212-z

Kim, J. J., \& Park, S. Y. (2016). Immediate effects of the trunk stabilizing exercise on static balance parameters in double-leg and one-leg stances. Journal of physical therapy science, 28(6), 1673-1675. https://doi.org/10.1589/jpts.28.1673

Lee, C. H., \& Sun, T. L. (2018). Evaluation of postural stability based on a force plate and inertial sensor during static balance measurements. Journal of physiological anthropology, 37(1), 27. https://doi.org/10.1186/s40101-018-0187-5

Mancilla S, Eladio, Valenzuela H, José, \& Escobar C, Máximo. (2015). Rendimiento en las pruebas «Timed Up and Go» y «Estación Unipodal» en adultos mayores chilenos entre 60 y 89 años. Revista médica de Chile, 143(1), 39-46. https: / / dx.doi.org/10.4067/S0034-98872015000100005

Marmamula, S., Barrenkala, N. R., Challa, R., Kumbham, T. R., Modepalli, S. B.,Yellapragada, R., Bhakki, M., Friedman, D. S., \& Khanna, R. C. (2020). Falls and visual impairment among elderly residents in 'homes for the aged' in India. Scientific reports, 10(1), 13389. https://doi.org/10.1038/s41598020-70066-2

Mesquita, L. S., de Carvalho, F. T., Freire, L. S., Neto, O. P., \& Zângaro, R.A. (2015). Effects of two exercise protocols on postural balance of elderly women: a randomized controlled trial. BMC geriatrics, 15, 61. https://doi.org/10.1186/ s12877-015-0059-3

Navalón Alcañiz, R., \& Martínez González-Moro, I. (2020).Valoración del grado de deterioro funcional y fragilidad en adultos mayores activos. Retos, 38, 576-581. https://doi.org/ $10.47197 /$ retos.v38i38.78252

Oliveira, M. R.,Vieira, E. R., Gil,A., Fernandes, K., Teixeira,D. C., Amorim, C. F., \& da Silva, R. A. (2018). One-legged stance sway of older adults with and without falls. PloS one, 13(9), e0203887. journal.pone.0203887

Osoba, M.Y., Rao,A. K., Agrawal, S. K., \& Lalwani, A. K. (2019). Balance and gait in the elderly: A contemporary review. Laryngoscope investigative otolaryngology, 4(1), 143-153. https: / /doi.org/10.1002/lio2.252

Paillard, T., \& Noé, F. (2015). Techniques and Methods forTesting the Postural Function in Healthy and Pathological Subjects. BioMed research international, 2015, 891390. https:/ /doi.org/10.1155/2015/891390
Pelosin, E., Bisio, A., Pozzo, T., Lagravinese, G., Crisafulli, O., Marchese, R.,Abbruzzese, G., \& Avanzino, L. (2018). Postural Stabilization Strategies to Motor Contagion Induced by Action Observation Are Impaired in Parkinson's Disease. Frontiers in neurology, 9, 105. https://doi.org/10.3389/ fneur.2018.00105

Pua,Y. H., Ong, P. H., Clark, R. A., Matcher, D. B., \& Lim, E. C. (2017). Falls efficacy, postural balance, and risk for falls in older adults with falls-related emergency department visits: prospective cohort study. BMC geriatrics, 17(1), 291. https:/ /doi.org/10.1186/s12877-017-0682-2

Puszczalowska-Lizis, E., Bujas, P., Jandzis, S., Omorczyk, J., \& Zak, M. (2018). Inter-gender differences of balance indicators in persons 60-90 years of age. Clinical interventions in aging, 13, 903-912. https://doi.org/10.2147/CIA.S157182

Riva, D., Fani, M., Benedetti, M. G., Scarsini,A., Rocca, F., \& Mamo, C. (2019). Effects of High-Frequency ProprioceptiveTraining on Single Stance Stability in Older Adults: Implications for Fall Prevention. BioMed research international, 2019, 2382747. https: / /doi.org/10.1155/2019/2382747

Saftari, L. N., \& Kwon, O. S. (2018). Ageing vision and falls: a review. Journal of physiological anthropology, 37(1), 11. https: / / doi.org/10.1186/s40101-018-0170-1

Salazar Martínez, C., Villar Ortega, M., Párraga Montilla, J.A., \& Moreno, R. (2015). Efectos de la utilización de los videojuegos en un programa de mejora del equilibrio en mujeres de 60 años o más. Retos, 17, 93-95. https://doi.org/10.47197/ retos.v0i17.34682

Sampedro Molinuevo, J., Meléndez Ortega, A., \& Ruiz Solano, P. (2015). Análisis comparativo de la relación entre el número de caídas anual y baterías de pruebas de equilibrio y agilidad en personas mayores. Retos, 17, 115-117. https://doi.org/ 10.47197/retos.v0i17.34689

Saüch, G., Castañer, M., \& Hileno, R. (2015).Valorar la capacidad de equilibrio en la tercera edad. Retos, 23, 48-50. https:// doi.org/10.47197/retos.v0i23.34567

Semak, M.R., Schwartz, J., \& Heise, G. (2020). Examining Human Unipedal Quiet Stance: Characterizing Control through Jerk. Computational and mathematical methods in medicine, 2020, 5658321. https://doi.org/10.1155/2020/5658321

Springer, B. A., Marin, R., Cyhan, T., Roberts, H., \& Gill, N.W. (2007). Normative values for the unipedal stance test with eyes open and closed. Journal of geriatric physical therapy (2001), 30(1), 8-15. https://doi.org/10.1519/00139143200704000-00003

Toosizadeh, N., Ehsani, H., Miramonte, M., \& Mohler, J. (2018). Proprioceptive impairments in high fall risk older adults: the effect of mechanical calf vibration on postural balance. Biomedical engineering online, 17(1), 51. https: / / doi.org/10.1186/s12938-018-0482-8

Yamagata, M., Ikezoe, T., Kamiya, M., Masaki, M., \& Ichihashi, N. (2017). Correlation between movement complexity during static standing and balance function in institutionalized older adults. Clinical interventions in aging, 12, 499-503. https:// doi.org/10.2147/CIA.S132425 